\title{
CT colonography: inverse-consistent symmetric registration of prone and supine inner colon surfaces
}

\author{
Holger R. Roth ${ }^{a}$, Jamie R. McClelland ${ }^{a}$, Marc Modat ${ }^{a}$, Thomas E. Hampshire ${ }^{a}$, Darren J. \\ Boone $^{b}$, Emma Helbren ${ }^{b}$, Andrew Plumb ${ }^{b}$, Mingxing $\mathrm{Hu}^{a}$, Sebastien Ourselin ${ }^{a}$, Steve \\ Halligan $^{b}$ and David J. Hawkes ${ }^{a}$ \\ ${ }^{a}$ Centre for Medical Image Computing, University College London, UK \\ ${ }^{b}$ Centre for Medical Imaging, University College London, London, UK
}

\begin{abstract}
CT colonography interpretation is difficult and time-consuming because fecal residue or fluid can mimic or obscure polyps, leading to diagnostic errors. To compensate for this, it is normal practice to obtain CT data with the patient in prone and supine positions. Repositioning redistributes fecal residue and colonic gas; fecal residue tends to move, while fixed mural pathology does not.

The cornerstone of competent interpretation is the matching of corresponding endoluminal locations between prone and supine acquisitions. Robust and accurate automated registration between acquisitions should lead to faster and more accurate detection of colorectal cancer and polyps. Any directional bias when registering the colonic surfaces could lead to incorrect anatomical correspondence resulting in reader error. We aim to reduce directional bias and so increase robustness by adapting a cylindrical registration algorithm to penalize inverse-consistency error, using a symmetric optimization.

Using 17 validation cases, the mean inverse-consistency error was reduced significantly by $86 \%$, from $3.3 \mathrm{~mm}$ to $0.45 \mathrm{~mm}$. Furthermore, we show improved alignment of the prone and supine colonic surfaces, evidenced by a reduction in the mean-of-squared-differences by $43 \%$ overall. Mean registration error, measured at a sparse set of manually selected reference points, remained at the same level as the non-symmetric method (no significant differences). Our results suggest that the inverse-consistent symmetric algorithm performs more robustly than non-symmetric implementation of B-spline registration.
\end{abstract}

Keywords: virtual colonoscopy, CT colonography, prone-to-supine registration, non-rigid image registration, inverse-consistent registration, conformal mapping, computer-aided diagnosis and interventions

\section{INTRODUCTION}

Robust and accurate registration of prone and supine colonic surfaces acquired during CT colonography may lead to faster and more precise detection of colorectal cancer and polyps. Any directional bias when registering the colonic surfaces could lead to incorrect anatomical correspondence resulting in diagnostic errors. Despite this, non-rigid registration methods are often implemented asymmetrically, which could influence the registration result negatively. We aim to reduce directional bias and so increase robustness by adapting a cylindrical registration algorithm to be both symmetric and inverse-consistent.

The problem of establishing spatial correspondence between the full colonic surface extracted from prone and supine CT colonography acquisitions is a challenging one. Several algorithms have been proposed to align both surfaces (e.g. $[9,15,19,13])$. All these methods assume that registration is being performed with one image (e.g. the prone) as the transformed or "floating" image and the other image (e.g. the supine) as the "reference" image. Image registration then finds spatial correspondences between the reference and floating image. However, computing these correspondences in just one direction might bias the registration result: i.e. such computation might result in different spatial correspondences depending on the order of input images. Assuming there is a unique anatomical correspondence between the prone and supine surface, this is undesirable. The result should

Further author information: Send correspondence to Holger R. Roth: E-mail: h.roth@ucl.ac.uk 
ideally be identical when registering the prone to the supine colonic surface or the converse. Inverse-consistent symmetry in the registration result, which is independent of the order of input images is preferable.

The amount of difference depending on the direction of registration can be measured by the inverse consistency error $(I C E)$. This measure was proposed by Christensen et al. [3] as an important criterion for evaluation of non-rigid registration methods. A prone-to-supine registration method which aims to minimize $I C E$ could lead to improved registration accuracy and robustness by providing inversely consistent anatomical correspondence. By constraining inverse-consistency in the registration, false local minima in the objective function might be reduced. In such cases the registration might achieve better alignment using an inverse-consistent symmetric approach. Using both forward (prone-to-supine) and backward (supine-to-prone) directions for registration may encourage convergence towards the correct result. Symmetric image registration has been shown to reduce local minima in the objective function and generally improves registration quality $[2,17]$.

\section{RELATED WORK}

Recent work that aims to remove directional bias in non-rigid image registration algorithms includes Symmetric Normalization (SyN) [2] from the Advance Normalization Tools (ANTs*) package and the approach proposed by Vercauteren et al. [17], using demons-based registration. Bias introduced by the direction of registration was recently shown to cause unrealistic estimation of statistical power when quantifying longitudinal atrophy in structural MRI images [16]. We showed previously that using robustly matched features to initialise the well-known non-rigid B-spline registration method [14] can reduce the inverse consistency error [12]. This generally increases the quality of the registration result. However, the standard B-spline registration algorithm is not inverse-consistent and could therefore still bias the result negatively towards direction of registration. In order to overcome this limitation, Christensen and Johnson [4] proposed an inverse-consistent image registration method using a 3-D Fourier series representation for parametrization of the forward and backward transformations [1]. Feng et al. [5] modified the B-spline registration to minimize the inverse consistency error by optimizing forward and backward transformations concurrently. However, their implementation does not ensure one-to-one correspondence. Preservation of one-to-one correspondence was incorporated by Modat et al. [10] using a Jacobian-based constraint. We follow this approach based on B-spline registration but modify the deformation to occur in cylindrical space, taking advantage of the anatomical topology specific to the colon. We concurrently optimise the prone-to-supine and supine-to-prone transformations to be inverse consistent and symmetric. Furthermore, we penalize both transformations against folding and therefore encourage one-to-one mappings. Assessment of our implementation is performed using a set of fully distended cases as well as cases exhibiting local colonic collapse.

\section{METHODS}

\subsection{Registration of prone and supine colonic surfaces}

Our prone-to-supine registration method involves the conformal mapping of both colon surfaces derived from the endoluminal segmentations to cylindrical representations. This mapping simplifies this challenging 3D registration problem to the problem of cylindrical registration in 2D. Initialization between the cylindrical images is provided by robust feature matches of haustral folds between both views using a hierarchical B-spline approximation as proposed by Hampshire et al. [6, 7]. A non-rigid intensity-based registration based on the well-accepted B-spline registration method is then used to establish correspondence in this cylindrical domain [13]. This is achieved by minimizing the mean-of-squared differences $(M S D)$ between both cylindrical images. Each cylindrical image contains curvature information of the local colon surface, measured using shape index $(S I)$, which has also been used to detect colonic polyps by computer-aided detection (CAD) methods for CT colonography images [18].

In the case of local colonic under-distention in either prone and/or supine images, the collapsed region can be ignored during non-rigid registration when computing the $S S D$ similarity measure. This is an important feature of prone-to-supine registration algorithms as luminal collapse is encountered commonly in clinical practice [8].

\footnotetext{
*http://picsl.upenn.edu/ANTS
} 


\subsection{Symmetric prone-to-supine transformation model}

We implemented a symmetric formulation of a non-rigid B-spline image registration method in cylindrical space in order to align the cylindrical representations of the prone and supine endoluminal surfaces. Our cylindrical implementation is based on the open-source ${ }^{\dagger}$ software by Modat et al. [10]. A symmetric similarity cost function measures the mean-of-squared-differences $(M S D)$ between the prone and supine cylindrical images $P$ and $S$, using both the prone-to supine transformation $\mathbf{T}_{\mathrm{ps}}$ and the supine-to-prone transformation $\mathbf{T}_{\mathrm{sp}}$ simultaneously. This principle is illustrated in Fig. 1, where the colour indicates local $S I$ values. Inverse consistency of the transformation is encouraged by adding an appropriately weighted penalty term to the optimization function (see Equation 2).
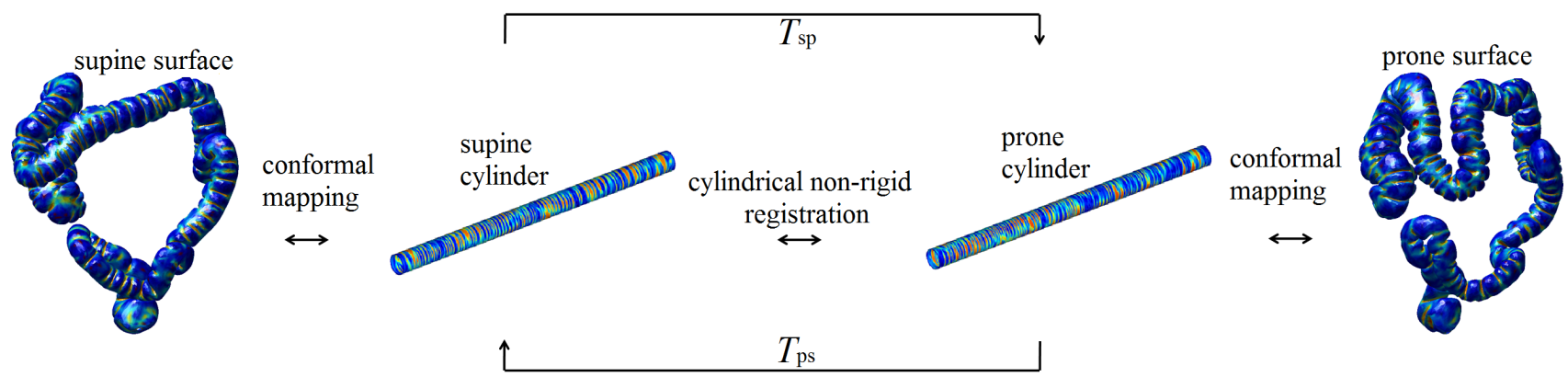

Figure 1. Symmetric prone-to-supine transformation model. The colour indicates the local shape indices.

A symmetric similarity cost function measures the mean-of-squared differences $(M S D)$ between the prone and supine cylindrical images $P$ and $S$, using both the prone-to-supine transformation $\mathbf{T}_{\mathrm{ps}}$ and the supine-to-prone transformation $\mathbf{T}_{\mathrm{sp}}$ simultaneously:

$$
M S D_{\mathrm{Sym}}=-\frac{1}{N_{S}} \sum_{\vec{x} \forall S}\left[S(\vec{x})-P\left(\mathbf{T}_{\mathrm{ps}}(\vec{x})\right)\right]^{2}-\frac{1}{N_{P}} \sum_{\vec{x} \forall P}\left[P(\vec{x})-S\left(\mathbf{T}_{\mathrm{sp}}(\vec{x})\right)\right]^{2}
$$

Both transformations are implemented in cylindrical space. In an ideal case, these transformations are the inverse of each other, e.g. $\mathbf{T}_{\mathrm{ps}}=\mathbf{T}_{\mathrm{sp}}^{-1}$ and $\mathbf{T}_{\mathrm{sp}}=\mathbf{T}_{\mathrm{ps}}^{-1}$. However, this symmetric similarity measure alone does not ensure that the registration is inverse-consistent. Additionally, one needs to modify the optimization function to include a penalty term that encourages inverse consistency of both transformations. We follow the approach of Feng et al. [5] using compositions of $\mathbf{T}_{\mathrm{ps}}$ and $\mathbf{T}_{\mathrm{sp}}$ and add

$$
\mathcal{P}_{\mathrm{IC}}=\sum_{\vec{x} \forall S}\left\|\mathbf{T}_{\mathrm{sp}}\left(\mathbf{T}_{\mathrm{ps}}(\vec{x})\right)\right\|^{2}+\sum_{\vec{x} \forall P}\left\|\mathbf{T}_{\mathrm{ps}}\left(\mathbf{T}_{\mathrm{sp}}(\vec{x})\right)\right\|^{2}
$$

to the objective function being optimised. Furthermore, we include two more symmetric penalty terms $\mathcal{P}_{\mathrm{BE}}$ and $\mathcal{P}_{\text {Jac }}$ in order to promote realistic deformations. The first term $\mathcal{P}_{\mathrm{BE}}$ causes a smooth transformation based on bending energy:

$$
\begin{aligned}
\mathcal{P}_{\mathrm{BE}} & =\frac{1}{N} \sum_{\vec{x} \forall S}\left(\left|\frac{\partial^{2} \mathbf{T}_{\mathrm{sp}}(\vec{x})}{\partial x^{2}}\right|^{2}+\left|\frac{\partial^{2} \mathbf{T}_{\mathrm{sp}}(\vec{x})}{\partial y^{2}}\right|^{2}+2\left|\frac{\partial^{2} \mathbf{T}_{\mathrm{sp}}(\vec{x})}{\partial x y}\right|^{2}\right) \\
& +\frac{1}{N} \sum_{\vec{x} \forall P}\left(\left|\frac{\partial^{2} \mathbf{T}_{\mathrm{ps}}(\vec{x})}{\partial x^{2}}\right|^{2}+\left|\frac{\partial^{2} \mathbf{T}_{\mathrm{ps}}(\vec{x})}{\partial y^{2}}\right|^{2}+2\left|\frac{\partial^{2} \mathbf{T}_{\mathrm{ps}}(\vec{x})}{\partial x y}\right|^{2}\right) .
\end{aligned}
$$

The inverse-consistency penalty term $\mathcal{P}_{\mathrm{IC}}$ alone does not guarantee folding-free transformations as the inverseconsistency error is constrained but will not be zero. Hence, we also add a second Jacobian-based penalty term

\footnotetext{
${ }^{\dagger} \mathrm{http}: / /$ sourceforge.net/projects/niftyreg
} 
$\mathcal{P}_{\text {Jac }}$ in order to encourage one-to-one mapping by preventing folding as in [10]:

$$
\mathcal{P}_{\mathrm{Jac}}=\frac{1}{N} \sum_{\vec{x} \forall S}\left[\log \left(\operatorname{det}\left(\operatorname{Jac}\left(\mathbf{T}_{\mathrm{sp}}\right)\right)\right)\right]^{2}+\frac{1}{N} \sum_{\vec{x} \forall P}\left[\log \left(\operatorname{det}\left(\operatorname{Jac}\left(\mathbf{T}_{\mathrm{ps}}\right)\right)\right)\right]^{2}
$$

Therefore, the function being optimised in the inverse-consistent symmetric registration scheme is:

$$
\mathcal{O}\left(P, S ; \mu_{p s}, \mu_{s p}\right)=(1-\alpha-\beta-\gamma) \times M S D_{\mathrm{Sym}}+\alpha \times \mathcal{P}_{\mathrm{BE}}+\beta \times \mathcal{P}_{\mathrm{Jac}}+\gamma \times \mathcal{P}_{\mathrm{IC}}
$$

where $\mu_{p s}$ and $\mu_{s p}$ are the control point positions defining the transformations $\mathbf{T}_{\mathrm{ps}}$ and $\mathbf{T}_{\mathrm{sp}}$. The parameters $\alpha$, $\beta$ and $\gamma$ are weights that control the influence of each penalty term. $\alpha+\beta+\gamma$ need to be less than 1 .

\subsection{Optimization}

We use a conjugate gradient ascent optimization in order to maximize the objective function of Equation 5. For optimization, the gradients of $\mathcal{O}$ with respect to the control points $\mu_{p s}$ and $\mu_{s p}$ are required:

$$
\frac{\partial \mathcal{O}\left(P, S ; \mu_{p s}, \mu_{s p}\right)}{\partial \mu_{p s}} \text { and } \frac{\partial \mathcal{O}\left(P, S ; \mu_{p s}, \mu_{s p}\right)}{\partial \mu_{s p}}
$$

The analytical derivatives of the $M S D$ similarity measure, bending energy $\mathcal{P}_{\mathrm{BE}}$ and the Jacobian-based penalty term $\mathcal{P}_{\text {Jac }}$ have been defined in the literature [11]. The derivative of the inverse-consistency error penalty term $\mathcal{P}_{\text {IC }}$ can be computed using a two step approach as in [10]. Firstly, the ICE derivatives are computed at each voxel position and then the derivatives are estimated at the control point positions of $\mu_{p s}$ and $\mu_{s p}$. This is implemented by computing four displacement fields using composition:

$$
\begin{array}{ll}
-D 1_{P}(x)=x-\mathbf{T}_{\mathrm{ps}}\left(\mathbf{T}_{\mathrm{sp}}(x)\right) & \text { where } x \in P \\
-D 2_{P}(x)=x-\mathbf{T}_{\mathrm{sp}}\left(\mathbf{T}_{\mathrm{ps}}(x)\right) & \text { where } x \in P \\
-D 1_{S}(x)=x-\mathbf{T}_{\mathrm{ps}}\left(\mathbf{T}_{\mathrm{sp}}(x)\right) & \text { where } x \in S \\
-D 2_{S}(x)=x-\mathbf{T}_{\mathrm{sp}}\left(\mathbf{T}_{\mathrm{ps}}(x)\right) & \text { where } x \in S
\end{array}
$$

A cubic B-spline kernel is then convolved with the computed displacements fields $D 1_{P}$ and $D 2_{P}$ in order to produce a cubic B-spline parametrization of the transformation $\mathbf{T}_{\mathrm{ps}}$ while ensuring a cylindrical transformation. The same is computed for $\mathbf{T}_{\mathrm{sp}}$ using the displacements fields $D 1_{S}$ and $D 2_{S}$. The gradient information at each control point position $\mu_{p s}$ and $\mu_{s p}$ can then be estimated using linear interpolation as in [10].

\section{RESULTS}

Registration parameters were tuned empirically on 8 well-prepared prone/supine CTC cases based on visual assessment and assessment of registration error. This resulted in a $73 \%$ reduction in mean $I C E$, while keeping similar registration accuracy compared to a standard asymmetric registration. Tuning resulted in the registration weights $\alpha\left(\mathcal{P}_{B E}\right), \beta\left(\mathcal{P}_{J a c}\right)$ and $\gamma\left(\mathcal{P}_{I C}\right)$ in the objective function (5) being set to $1 e^{-5}, 1 e^{-5}$ and $1 e^{-3}$, respectively. We found that these values for $\beta$ and $\gamma$ performed better in combination with the inverse-consistency penalty $\left(\mathcal{P}_{I C}\right)$, (using visual assessment), than the previously used weights of $1 e^{-4}$ and $1 e^{-4}$ for $\alpha$ and $\beta$ respectively (e.g. as used in [13]).

The following Table (1) shows the mean inverse consistency errors $(\overline{I C E})$ before $(1)$, and after $(2)$, penalizing for inverse consistency error using the symmetric registration approach in 17 prone/supine CTC sets (not used for tuning), of which five were exhibiting local colonic luminal collapse and four were excluded from previous studies due to marked differences in local distension and therefore different surface features (see [13] for more details). Furthermore, registration accuracy is measured using 1743 pairs of reference points. These reference points were manually selected between corresponding haustral folds by three experienced observers (two radiologists (EH, AP) and one computer scientist (TH)) in consensus using virtual 3D fly-through renderings for navigation through the prone and supine acquisitions; leading to 103 references points per case on average [6, 7]. These reference points enable the computation of a fold registration error $(F R E)$, measured as the Euclidean distance between corresponding points. 
In the validation set, the mean inverse-consistency error $(\overline{I C E})$ was reduced by $86 \%$ from $3.3( \pm 2.8) \mathrm{mm}$ to $0.45( \pm 0.16) \mathrm{mm}$ using the proposed symmetric formulation. This reduction of $\overline{I C E}$ is significant $(\mathrm{p}<0.001$, using a Related Samples Wilcoxon Signed Rank as for all tests of statistical significance applied in this study.).

The total mean FRE stayed at a similar value with 6.6 ( \pm 3.0$) \mathrm{mm}$ when compared to the previous value of $6.0( \pm 1.9) \mathrm{mm}$. This change in registration accuracy was not significant for the 17 validation data sets $(\mathrm{p}=$ 0.78). However, the registration error was improved significantly in the set of eight well-distended cases from 4.9 $( \pm 0.8) \mathrm{mm}$ to $4.4( \pm 0.7) \mathrm{mm}(\mathrm{p}=0.017)$. Nevertheless, the total registration error is slightly worse because of increased registration errors in the cases with a region of local collapse (patients 9-13) or large differences in distension (patients 14-17).

Table 1. Mean inverse consistency errors $(\overline{I C E})$, registration accuracy $(\overline{F R E})$ and mean-of-squared-differences $(M S D)$ without (1) and with (2) using the symmetric approach for the validation set of 17 CTC cases. The locations of where the colon is collapsed are given (DC: descending colon, SC: sigmoid colon).

\begin{tabular}{|c|c|c|c|c|c|c|c|c|}
\hline Patient & Collapse & Collapse & $\overline{\overline{I C E_{1}}}$ & $\overline{\overline{I C E_{2}}}$ & $\overline{\overline{F R E_{1}}}$ & $\overline{\overline{F R E_{2}}}$ & $M S D_{1}$ & $M S D_{2}$ \\
\hline $\begin{array}{l}\# \\
1\end{array}$ & $\begin{array}{l}\text { in prone } \\
\text { none }\end{array}$ & $\begin{array}{l}\text { in supine } \\
\text { none }\end{array}$ & $\begin{array}{r}{[\mathrm{mm}]} \\
2.2\end{array}$ & $\begin{array}{r}{[\mathrm{mm}]} \\
0.39\end{array}$ & $\begin{array}{r}{[\mathrm{mm}]} \\
6.4\end{array}$ & $\begin{array}{r}{[\mathrm{mm}]} \\
5.6\end{array}$ & $\begin{array}{r}{\left[\begin{array}{lll}\times & & \end{array}\right]} \\
1.8\end{array}$ & $\begin{array}{r}{\left[\begin{array}{ll}\times & e\end{array}\right]} \\
0.9\end{array}$ \\
\hline 2 & none & none & 1.7 & 0.34 & 5.4 & 4.8 & 2.0 & 0.9 \\
\hline 3 & none & none & 1.0 & 0.30 & 4.5 & 4.1 & 1.8 & 0.8 \\
\hline 4 & none & none & 1.4 & 0.30 & 4.3 & 3.9 & 2.1 & 1.0 \\
\hline 5 & none & none & 1.3 & 0.30 & 5.1 & 5.1 & 1.7 & 0.9 \\
\hline 6 & none & none & 1.1 & 0.30 & 3.6 & 3.1 & 2.2 & 1.1 \\
\hline 7 & none & none & 1.1 & 0.32 & 5.0 & 4.4 & 1.9 & 0.9 \\
\hline 8 & none & none & 1.2 & 0.32 & 4.9 & 4.5 & 1.7 & 1.0 \\
\hline Mean & & & 1.4 & 0.3 & 4.9 & 4.4 & 1.9 & 0.9 \\
\hline Std. dev. & & & 0.4 & 0.0 & 0.8 & 0.7 & 0.2 & 0.1 \\
\hline 9 & $1 \times \mathrm{DC}$ & $1 \times \mathrm{DC}$ & 2.9 & 0.62 & 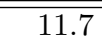 & 12.6 & 2.5 & 1.5 \\
\hline 10 & $1 \times \mathrm{DC}$ & $1 \times \mathrm{DC}$ & 2.9 & 0.35 & 5.7 & 9.3 & 2.4 & 1.3 \\
\hline 11 & none & $1 \times \mathrm{SC}$ & 11.0 & 0.68 & 5.0 & 7.3 & 2.5 & 2.1 \\
\hline 12 & $3 \times(\mathrm{DC}, \mathrm{SC})$ & none & 2.4 & 0.54 & 5.9 & 5.6 & 3.0 & 1.4 \\
\hline 13 & none & $1 \times \mathrm{DC}$ & 2.3 & 0.45 & 5.4 & 5.0 & 2.9 & 1.7 \\
\hline Mean & & & 4.3 & 0.5 & 6.7 & 7.9 & 2.7 & 1.6 \\
\hline \multirow[t]{2}{*}{ Std. dev. } & & & 3.7 & 0.1 & 2.8 & 3.1 & 0.3 & 0.3 \\
\hline & & Previously & $\overline{\text { excluded }}$ & & & & & \\
\hline 14 & none & none & 4.6 & 0.85 & 9.1 & 13.6 & 2.9 & 1.7 \\
\hline 15 & none & none & 6.2 & 0.58 & 7.8 & 7.3 & 3.0 & 1.6 \\
\hline 16 & none & none & 7.6 & 0.46 & 5.8 & 8.7 & 2.2 & 1.3 \\
\hline 17 & none & none & 5.7 & 0.55 & 6.8 & 6.8 & 2.5 & 1.3 \\
\hline Mean & & & 6.0 & 0.6 & 7.4 & 9.1 & 2.7 & 1.5 \\
\hline \multirow[t]{2}{*}{ Std. dev. } & & & 1.2 & 0.2 & 1.4 & 3.1 & 0.4 & 0.2 \\
\hline & & $\overline{~ T o t a l}$ & & & & & & \\
\hline Mean & & & 3.3 & 0.45 & 6.0 & 6.6 & 2.3 & 1.3 \\
\hline Std. dev. & & & 2.8 & 0.16 & 1.9 & 3.0 & 0.5 & 0.4 \\
\hline
\end{tabular}

The advantage of using an inverse-consistent symmetric formulation however is illustrated in Fig. 2 via a small section of the endoluminal colonic surface of one patient where no reference standard was selected. The local squared-differences $(S D)$ between the floating and the reference image pixels intensities is displayed before (left) and after (right) penalizing for inverse-consistency. The vertical dimension of both images goes around the circumference of the colon, while the horizontal dimension goes along the length of the colon. The $M S D$ was reduced in each of the 17 validation sets, with a total $43 \%$ reduction from $2.3( \pm 0.5) \times 10^{-2}$ to $1.3( \pm 0.4)$ $\times 10^{-2}$. This is a significant reduction in $M S D(\mathrm{p}<0.001)$. 

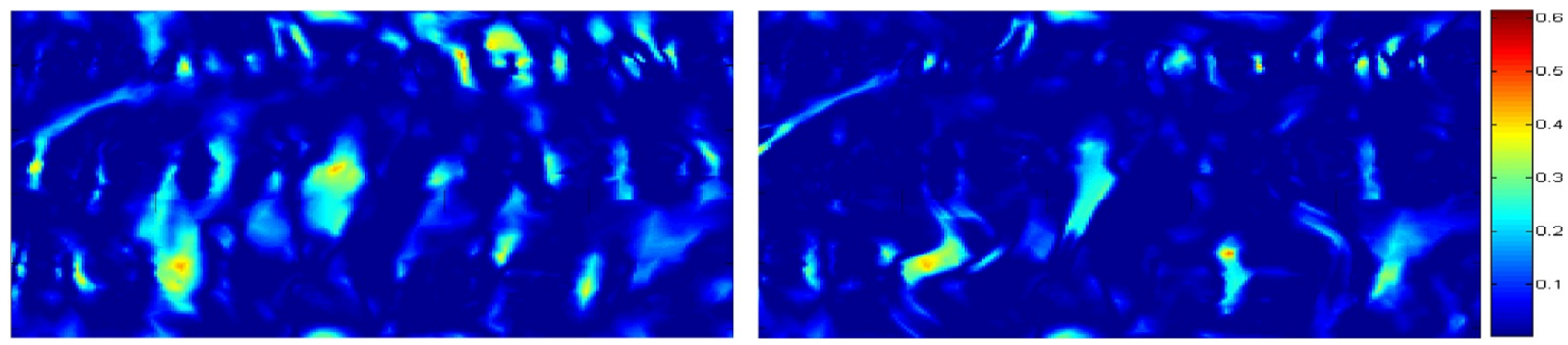

Figure 2. Local squared-differences $(S D)$ of registration result where no reference standard was provided. The $S D$ is measured between the floating and the reference image using the non-symmetric (left) and the inverse-consistent symmetric approach (right). Reduction in SD indicates an improved registration (right).

\section{CONCLUSIONS}

An extension to the previous work of Feng et al. [5], Modat et al. [10] and Roth et al. [13] is presented which enables inverse-consistent symmetric registration of prone and supine colonic endoluminal surfaces. Our transformations (both prone-to-supine and supine-to-prone) are parametrized, using a uniform cubic B-spline in cylindrical space. $M S D$ is used as a symmetric similarity measure. The inverse-consistent symmetric formulation reduces bias resulting from the direction of registration and has the potential to improve robustness and quality of the registration result.

We showed a large reduction of directional bias by implementing a symmetric non-rigid registration in cylindrical space that penalizes inverse-consistency error. The proposed registration method generates an improved visual alignment between the prone and supine colonic surfaces, as indicated by a highly significant reduction of $M S D$ between the prone and supine colon surfaces after registration. However, the registration error measured at a sparse set of manually selected reference points remained similar to the non-symmetric method.

Due to feasibility issues, the registration error can currently only be assessed with this sparse set of reference points as it is very challenging and time-consuming for radiologists to find corresponding locations between prone and supine positions manually. Therefore, only a limited region of the colon surface can be assessed with the reference standard we constructed. This is due to difficulties in finding accurate reference points when the appearance of the colon is largely different (e.g. differing distension or segmentation errors). This means that reference points are often not present in regions where the registration result has nevertheless improved visually (as indicated by a reduction in $M S D$, as shown for example in Fig. 2).

On the other hand, the fact that only well-distended cases show an improvement in registration error might indicate that a symmetric inverse-consistent registration is more dependent on good initialization (in our case using the method by Hampshire et al. [6, 7]). Cases with collapsed regions or cases that exhibit large differences in distension typically have less feature matches that can then be used for initialization. In the validation set used, cases with collapse or large differences in distension had an average 80 feature matches compared with an average 120 matches in the eight well-distended cases. If the initial alignment of the cylindrical intensity images is inadequate, a symmetric registration might 'pull' in different directions depending on the direction of registration. This will be penalized by the inverse-consistency term and can cause the registration to align features poorly in these regions.

This indicates that the inverse consistency weight $\gamma\left(\mathcal{P}_{I C}\right)$ was probably chosen too high for the cases exhibiting local endoluminal collapse or marked differences in distension - this is likely to be due to over-fitting to the welldistended cases that were used for tuning the registration parameters, these being the only ones available with reference standard. Although the total registration error is slightly worse, the difference is in fact negligible when compared to the uncertainty in identifying the exact locations of anatomically corresponding landmark points.

In conclusion, the highly significant improvement of inverse-consistency that has been demonstrated, together with the accompanying stability of registration accuracy, provides a more robust and reliable registration method - at least in well-prepared CTC cases. Information from both prone-to-supine and supine-to-prone directions 
helps to enforce convergence towards a more accurate solution if a good initialization is provided. More robust and accurate inverse-consistent symmetric registration in cylindrical space will likely facilitate improved interpretation of CT colonography.

\section{ACKNOWLEDGMENTS}

The authors gratefully acknowledge financial support for this work from the NIHR Program Grants for Applied Research: "Imaging diagnosis of colorectal cancer: Interventions for efficient and acceptable diagnosis in symptomatic and screening populations" (Grant No. RP-PG-0407-10338) and the EPSRC-CRUK Comprehensive Cancer Imaging Centre of UCL and KCL (Grant No. C1519AO).

\section{References}

[1] Amit, Y. (1994). A nonlinear variational problem for image matching. SIAM Journal on Scientific Computing 15(1), 207-224.

[2] Avants, B., C. Epstein, M. Grossman, and J. Gee (2008). Symmetric diffeomorphic image registration with cross-correlation: Evaluating automated labeling of elderly and neurodegenerative brain. Medical image analysis 12(1), 26-41.

[3] Christensen, G., X. Geng, J. Kuhl, J. Bruss, T. Grabowski, I. Pirwani, M. Vannier, J. Allen, and H. Damasio (2006). Introduction to the non-rigid image registration evaluation project (nirep). Biomedical Image Registration 4057, 128-135.

[4] Christensen, G. and H. Johnson (2001, july). Consistent image registration. Medical Imaging, IEEE Transactions on 20(7), $568-582$.

[5] Feng, W., S. Reeves, T. Denney, S. Lloyd, L. Dell'Italia, and H. Gupta (2009). A new consistent image registration formulation with a b-spline deformation model. In Biomedical Imaging: From Nano to Macro, 2009. ISBI'09. IEEE International Symposium on, pp. 979-982. IEEE.

[6] Hampshire, T., H. Roth, D. Boone, G. Slabaugh, S. Halligan, and D. Hawkes (2012). Prone to supine ct colonography registration using a landmark and intensity composite method. Abdominal Imaging. Computational and Clinical Applications, 1-9.

[7] Hampshire, T., H. Roth, E. Helbren, A. Plumb, D. Boone, G. Slabaugh, S. Halligan, and D. Hawkes (2013). Automated registration for ct colonography using a markov random field composite method. Medical Image Analysis - submitted.

[8] Hara, A., M. Kuo, M. Blevins, M. Chen, J. Yee, A. Dachman, C. Menias, B. Siewert, J. Cheema, R. Obregon, et al. (2011). National ct colonography trial (acrin 6664): comparison of three full-laxative bowel preparations in more than 2500 average-risk patients. Am. J. Roentgenol. 196(5), 1076-1082.

[9] Huang, A., D. Roy, R. Summers, M. Franaszek, N. Petrick, J. Choi, and P. Pickhardt (2007). Teniae colibased circumferential localization system for CT colonography: Feasibility study. Radiology 243(2), 551-560.

[10] Modat, M., M. Cardoso, P. Daga, D. Cash, N. Fox, and S. Ourselin (2012). Inverse-consistent symmetric free form deformation. Biomedical Image Registration 7359, 79-88.

[11] Modat, M., G. Ridgway, Z. Taylor, M. Lehmann, J. Barnes, D. Hawkes, N. Fox, and S. Ourselin (2010). Fast free-form deformation using graphics processing units. Comput. Meth. Prog. Bio. 98(3), 278-284.

[12] Roth, H., T. Hampshire, J. McClelland, M. Hu, D. Boone, G. Slabaugh, S. Halligan, and D. Hawkes (2012). Inverse consistency error in the registration of prone and supine images in ct colonography. Abdominal Imaging. Computational and Clinical Applications 7029, 1-7. 
[13] Roth, H. R., J. R. McClelland, D. J. Boone, M. Modat, M. J. Cardoso, T. E. Hampshire, M. Hu, S. Punwani, S. Ourselin, G. G. Slabaugh, S. Halligan, and D. J. Hawkes (2011). Registration of the endoluminal surfaces of the colon derived from prone and supine ct colonography. Medical Physics 38(6), 3077-3089.

[14] Rueckert, D., L. Sonoda, C. Hayes, D. Hill, M. Leach, and D. Hawkes (1999). Nonrigid registration using free-form deformations: Application to breast mr images. IEEE Trans. Med. Imaging 18(8), 712-721.

[15] Suh, J. and C. Wyatt (2009). Deformable registration of supine and prone colons for computed tomographic colonography. J. Comput. Assist. Tomogr. 33(6), 902-911.

[16] Thompson, W., D. Holland, et al. (2011). Bias in tensor based morphometry stat-roi measures may result in unrealistic power estimates. Neuroimage $57(1), 1$.

[17] Vercauteren, T., X. Pennec, A. Perchant, and N. Ayache (2008). Symmetric log-domain diffeomorphic registration: A demons-based approach. Medical Image Computing and Computer-Assisted InterventionMICCAI 2008 5241, 754-761.

[18] Yoshida, H. and J. Nappi (2002). Three-dimensional computer-aided diagnosis scheme for detection of colonic polyps. IEEE Trans. Med. Imaging 20(12), 1261-1274.

[19] Zeng, W., J. Marino, K. Chaitanya Gurijala, X. Gu, and A. Kaufman (2010). Supine and prone colon registration using quasi-conformal mapping. IEEE Trans. Vis. Comput. Graphics 16(6), 1348-1357. 